\title{
HACIA UNA TEORÍA SOCIOLÓGICA ABIERTA Y ANALITICA DE LA TRANSICIÓN POLÍTICA ${ }^{1}$
}

\section{Towards an open and analytical sociological theory of political transitions}

\author{
Alfonso Pérez-Agote* \\ TRANSOC, UCM
}

\section{Keywords}

Transition Modernization Civil society Public religious space

ABSTRACT: This paper aims to examine in depth, in order to avoid them, some of the consequences of the fact that the social sciences began to develop in Western European countries during periods in which the construction of modern, national, representative and democratic states was being sought. If these sciences adopted their theoretical and empirical profile associated with this project of nationalization and democratization of the state, reaching a prophetic dimension that draws the future of the populations of the planet, there is room for other sociologies, more sensitive to the diversity of modernizing projects, which seek to get rid of this prophetic character without losing the conceptual and theoretical richness of our classics. In this sense, this text problematizes the idea of political transition, rethinks the implicit question embedded, as any transition, in it (what is the relationship between power and the individuals subjected to it?) and thus seeks to implement a more open and analytical and less prophetic and evaluative social theory. The work ends by referring to the analysis of the transition in the West African countries of former French colonization, where the advent of a national democratic state does not seem feasible, but where creative processes of relationship between politics, religion and society are taking place.

\begin{abstract}
Palabras clave
Transición Modernización Sociedad civil Espacio público religioso

RESUMEN: Este trabajo pretende profundizar, para evitarlas, en algunas consecuencias de que las ciencias sociales comenzaran a desarrollarse en los países europeos occidentales en periodos en los que se pretendía la construcción de estados modernos, nacionales, representativos, y democráticos. Si estas ciencias adoptaron su perfil teórico y empírico asociado a ese proyecto de nacionalización y democratización del Estado, alcanzando una dimensión profética que dibuja el futuro de las poblaciones del planeta, caben otras sociologías, que más sensibles a la diversidad de proyectos modernizadores buscan deshacerse de ese carácter profético sin perder por ello la riqueza conceptual, teórica, de nuestros clásicos. En esa apuesta, este texto problematiza la idea de transición política, se replantea la pregunta implícita que lleva incrustada, como toda transición (¿cuál es la relación entre el poder y los individuos sometidos a este?) y procura, así, poner en marcha una teoría social más abierta y analítica y menos profética y valorativa. El trabajo termina refiriéndose al análisis de la transición en los países de África del oeste de anterior colonización francesa, en los que no parece factible la llegada de un Estado democrático nacional, pero se dan procesos creativos de relación entre política, religión y sociedad.
\end{abstract}

1 Este artículo ha sido producido en el marco del programa de investigación "Sortir de la violence» (SOV) financiado por la Agence Nationale de la Recherche francesa (Project ANR-16-CE39-001).

\footnotetext{
* Correspondencia a / Correspondence to: Alfonso Pérez-Agote. Universidad Complutense de Madrid. Campus de Somosaguas, s/n (28223 Pozuelo de AlarcónMadrid) - pagotea@yahoo.com - http://orcid.org/0000-0003-0681-8957.

Cómo citar / How to cite: Pérez-Agote, Alfonso (2021). «Hacia una teoría sociológica abierta y analítica de la transición política». Papeles del CEIC, vol. 2021/1, papel 239, 1-20. (http://dx.doi.org/10.1387/pceic.22064)
}

ISSN 1695-6494 / ㄷ 2021 UPV/EHU 
En general, cuando abordamos el tema de la transición política, los politólogos y los sociólogos lo hacemos bajo un supuesto que aparece más o menos explícito; en el fondo, pensamos en el proceso a través del cual un país cambia desde una forma autoritaria de gobierno hasta la consolidación de una fórmula democrática, entendida esta en el sentido de su semejanza con la forma adoptada históricamente por los llamados países occidentales. Esta constatación aparentemente simple me lleva a poner de relieve su compleja significación en tres ámbitos diferentes, íntimamente relacionados entre sí.

En primer lugar, es preciso analizar el proceso histórico de modernización de los países occidentales como proceso en el que se inicia la creación de una forma específica de relación entre el poder político y la población sometida a ese poder; el objetivo específico principal de la actividad política está referido a la solución de los problemas y carencias de esa población; se plantea así la cuestión de la representación política; los políticos representan a la población, son elegidos democráticamente por ella y son responsables ante ella en el ejercicio de su cargo; el objetivo de la actividad política está en la población que se convierte así en una sociedad, en una realidad social organizada por y en el Estado; esta población tiene conciencia de pertenencia a esa sociedad que es la nación; a esta conciencia colectiva de pertenencia se accede en un proceso de génesis y otro de posterior difusión de esa idea a través de diferentes mecanismos políticos y sociales. Este complejo proceso de modernización, presupone, por regla general, la existencia de un Estado, es decir, de un centro de poder establecido sobre un territorio físicamente definido.

En segundo lugar, la sociología es una ciencia que nace históricamente para el análisis de esta relación entre el poder político y la población; el proceso de modernización es un proyecto cuyo agente principal es el Estado, que trata de establecer esa nueva forma de gobierno y la sociología es uno de los medios útiles de reflexión sobre el proceso.

Pero, al mismo tiempo, y, en tercer lugar, esta ciencia adoptó una actitud profética prediciendo que en el futuro la humanidad irá progresivamente adoptando esa nueva forma de gobierno; esta dimensión profética menoscabó su capacidad analítica general, desdibujando el carácter histórico de la realidad social, empobreciendo así el carácter relativo, por ser históricos, de sus nociones y conceptos que son sus instrumentos analíticos.

La sociología histórica (Casanova, 2006) y la genética de los conceptos (Swidler, 1986; Asad, 2003) son las herramientas que algunos sociólogos usamos para tratar de desproveer de su carácter universalista, transhistórico, a los conceptos y las teorías derivadas del análisis de dichos procesos históricos. Estos conceptos y teorias pueden convertirse así en instrumentos analíticos útiles para el análisis de cualquier realidad social.

\section{EL ESTADO MODERNO DE EUROPA OCCIDENTAL}

La forma básica de diferenciación social, para Luhmann (1990), es la separación de la sociedad con relación a su entorno, centrando su reflexión en el entorno físico de la sociedad, dado que pretende abrir su reflexión a la cuestión ecológica, tan importante en nuestros dias. Pero se puede pensar también en un entorno social, compuesto por otras sociedades. Si pen- 
samos en la sociología histórica, esta ha sido esencial para comprender el proceso por el cual se van formando en Europa los Estados, llegándose a hablar del sistema europeo de Estados (Tilly, 1990). Desde este punto de vista es muy productiva la idea de representación política desarrollada por Voegelin (1968).

Para Voegelin (ibídem) existen dos maneras de comprender la representación política; en nuestra realidad social domina una forma, la llama elemental, que consiste en una descripción más o menos estricta de las instituciones de la democracia representativa. Sin embargo, en sentido teórico profundo, representación equivale a la específica articulación de la realidad social en orden a la posibilidad de actuar. La representación democrática sería una forma específica entre otras formas posibles. Fue esta forma específica la que se desarrolló en los procesos de modernización de los países europeos occidentales. La representación política, para Voegelin (ibídem: 65-67), es el origen de la primera ${ }^{2}$ diferenciación social, la organización interna para la acción: es el mismo proceso el que diferencia la sociedad del entorno y el que en el interior de esa sociedad se genera una parte, por ejemplo, un rex elegido para defenderse del enemigo, que se diferencia del resto de esa sociedad. La representación, en este sentido más científico, sería así el momento histórico de la aparición de un representante con capacidad de llevar a cabo una acción que compromete a toda la población, con lo cual se está fundando la sociedad como totalidad. Voegelin nos proporciona un esquema de cómo se produce en Europa ${ }^{3}$ el paso sucesivo por una serie de estadios de representación política que van desde unna primera fase [en la que] solo el rey es el representante del reino (...) hasta llegar a un límite en el que la pertenencia a la sociedad se ha llegado a articular políticamente hasta el último individuo y, por consiguiente, la sociedad llega a representarse a sí misma» (ibídem), que sería el momento democrático de la representación política. El orden político articula la sociedad, totalizándola, en tanto que sociedad política.

\subsection{Los tiempos previos y el surgimiento del Estado}

Para acercarme a los cambios históricos de la estructura política en los países occidentales voy a fijarme en dos elementos clave. El primero de ellos es de carácter objetivo, la concentración del poder en un centro que domina sobre un territorio concreto. El segundo es de carácter subjetivo, pues se refiere a la aceptación del poder, al sentido que el poder tiene para los gobernados; es el problema de la legitimidad, de cómo está socialmente considerado el vínculo político entre el que manda y los que obedecen.

En la época feudal, sobre los territorios que más tarde pasarán a ser territorios estatales, encontramos una serie de relaciones políticas de subordinación personal, ancladas fundamentalmente en las relaciones de vasallaje. Es decir, el poder político está disperso en una pluralidad de relaciones políticas; y estas son eminentemente personales, es decir, se trata de una legitimación personal del poder. El Estado es algo amorfo (Abercrombie, Hill y Turner, 1984) o, más bien, podemos decir que no existe.

2 El entorno puede ser físico o social, y la organización para la acción puede ser para la acción colectiva en relación con el entorno físico (la construcción de un dique para retener el agua) o con un entorno social (defensa, guerra, pacto).

3 Por supuesto que se pueden encontrar totalizaciones políticas —representaciones- en épocas más tempranas y en territorios alejados al contexto socio-histórico antedicho. El propio Voegelin (ibídem) cita ejemplos. 
La salida de la época feudal llevó consigo una serie de procesos bélicos entre quienes luego formarían los Estados europeos. El que Tilly (1990) denominó "sistema europeo de Estados», quedó plenamente configurado en la Paz de Westfalia (1648). Este proceso (en forma de bodas, herencias, guerras y pactos) fue el de construcción histórica de los elementos objetivos del Estado: el centro de poder con monopolio de la violencia física y el territorio delimitado por fronteras fijas.

En términos de la teoría de la diferenciación, lo que queda establecido en su momento fundacional es cada Estado en sus elementos objetivos, separándose de su entorno de otros Estados, porque existe un poder militar acumulado por un Rey que es así capaz de mantener la paz interna (derrotando a los otros posibles poderes militares) y de defender sus fronteras. Tilly (ibídem: 103-107) nos explica muy bien la importancia de los cambios en la esfera militar para la acumulación de ese poder por el Rey dentro de un territorio. La gran transformación en el ámbito militar posibilitó la creación de un ejército capaz de imponer la paz interna y de mantener la externa. En esos momentos primeros, el Estado, prácticamente, es el ejército.

En este periodo de concentración del poder militar-político en el rey ocupa un lugar central la génesis de la corte, como mecanismo de concentración. En La sociedad cortesana, Norbert Elias (1982) nos describe a la perfección los mecanismos a través de los cuales los monarcas europeos, acaban succionando el poder de la nobleza. Esta va dejando de tener poder en función de sus posesiones territoriales y lo va adquiriendo en función de su mayor cercanía con el Rey, con la Corte.

El viejo principio cuius regio eius religio fue la paradigmática expresión de la ley de homogeneización religiosa en el interior de los Estados. La Reforma fue el momento en que la máxima tuvo que aplicarse con concreción, en orden a producir la unificación religiosa de la población bajo la religión del soberano. La política diseñada por el rey, en principio, no tenía como objetivo el bienestar de la población. El rey era la esfera política y el dominio sobre su reino era patrimonial. Los súbditos existian, pero nadie pensaba en ellos como una unidad política. El periodo absolutista, el derecho divino de los reyes fue la condensación de esta forma de Estado: el poder del rey viene de Dios, el pueblo tiene la religión de su rey.

Más tarde empezaría el proceso de modernización por el cual ese resto de la realidad social comenzaría a pensarse como totalidad, como sociedad, como el objeto de la política. En el proceso posterior de democratización-nacionalización del Estado, sería necesaria la idea de nación, la nueva identidad colectiva en la que reside el poder. En substitución de la idea del derecho divino de los reyes. Y es en este posterior proceso cuando el Estado debe autonomizarse de la religión y de la Iglesia. En el centro de la cuestión estaba la idea de laicidad.

\subsection{Elementos analíticos del Estado moderno}

«El Estado es aquella comunidad humana que en el interior de un determinado territorio -el concepto de "territorio" es esencial a la definición- reclama para sí (con éxito) el monopolio de la coacción física legítima.» (Weber, 1969: 1056)

La definición de Estado diseñada por Weber en Economía y Sociedad (1969) es excelente desde un punto de vista analítico, aunque en rigor es una definición de Estado moderno, porque hace referencia concreta a la legitimidad - lo que implica la percepción que la población 
tiene del centro de poder al que está sujeta- - y a la existencia de una comunidad -lo que implica que en la población se da uun sentimiento subjetivo (afectivo o tradicional) de los partícipes de constituir un todo», según su propia definición de comunidad (ibídem: 33).

En principio Weber puede haber tomado el término comunidad en un sentido genérico de agrupación humana o en un sentido más estricto, ateniéndose a su definición de comunidad; en el primer caso, comunidad sería una forma imprecisa de demarcación de la realidad social; pero el segundo sentido es más congruente con el resto de la definición, porque precisamente el elemento que sigue, el territorio, es el elemento esencial de demarcación física de la realidad social. Y es más útil este sentido estricto porque, dicho rápida y gráficamente, permite introducir en la definición de Estado el problema de la nación y establecer en profundidad las relaciones entre ambos: no se puede confundir la nación con el Estado, pero no puede comprenderse el significado político moderno de nación sin referencia a un Estado pasado, existente en la actualidad o concebido como futuro a conseguir.

El Estado delimita objetivamente la realidad social; los habitantes de un determinado territorio están bajo el ámbito estatal en el sentido de que no depende su sujeción al Estado de que exista o no el sentimiento subjetivo de comunidad; este sentimiento es el que se da en el Estado moderno nacional, perfectamente conformado. El elemento territorio es, en principio, un elemento físico, geográficamente definido por las fronteras defendidas por la violencia estatal (otro elemento de la definición). También el territorio es algo simbólico, significante; pero esta dimensión subjetiva del territorio sería una dimensión del primer elemento, la comunidad: "quienes vivimos aquí, dentro de estas fronteras territoriales, formamos una comunidad, una "nación"»; también pueden darse casos más complicados, como el de los nacionalismos periféricos, que afirmando la existencia de una comunidad nacional dentro de un territorio parcial en relación al total del Estado existente, reclaman la creación de un Estado propio.

Además, es preciso tener en cuenta que el territorio es un elemento esencial a la definición porque el moderno Estado occidental abolió las vinculaciones políticas de tipo personal y, por tanto, la delimitación objetiva del vínculo político es necesariamente territorial (paso de un derecho personal a un derecho territorial, con respecto a su ámbito de validez) (Nisbet, 1970: capítulos 5 y 6).

Comunidad, elemento subjetivo, y territorio, elemento objetivo, son, por lo tanto, elementos diferentes e intimamente relacionados; puede darse coincidencia: en los habitantes del territorio físico existe el sentimiento de pertenencia a un todo; pero puede que no: en un territorio más pequeño que el estatal se da un sentimiento de pertenencia a la población de ese territorio parcial, excluyendo un sentimiento de dimensión estatal.

Otro de los elementos centrales de la noción weberiana de Estado es el «monopolio de la coacción física legítima». Es este un elemento complejo. La coacción física es el medio específico que utiliza el Estado en su actuación. «Desde el punto de vista de la consideración sociológica - dice Weber-, una asociación 'política' y en particular un 'Estado' no se pueden definir por el contenido de lo que hacen. En efecto, no existe apenas tarea alguna que una asociación política no haya tomado alguna vez en sus manos, ni tampoco puede decirse, por otra parte, que la política haya sido siempre exclusivamente propia de aquellas asociaciones que se designan como políticas, y hoy como Estados o que fueron históricamente las precursoras del Estado moderno" (Weber, 1969: 1056). Y Weber, citando a Trotsky, añade que "todo Estado se basa en la fuerza» (ibídem). Por tanto, para Weber, la coacción física es el elemento central y específico del Estado, por lo cual habla del monopolio de ese tipo de coacción. Es 
decir que, por un lado, nadie ni nada exterior al propio Estado puede ejercer la coacción física legítima en el interior de las fronteras estatales, y, por otro, monopolio interior, en el sentido de que nadie interior al Estado, pero no perteneciente al «aparato» de este, pueda ejercer coacción física legítima ${ }^{4}$. Esta es una deficiencia en la definición de Weber: no explicita la existencia de un centro diferenciado encargado del ejercicio de la coacción física.

Por otro lado, no se trata de simple monopolio de la coacción física, sino que, por un lado, se trata de que el Estado lo reclama para sí y, por otro, con «éxito». Es esta última una condición positiva, ya que se necesita la efectividad del monopolio, como condición de estabilidad del sistema político; $y$, por otra parte, es una forma expresa de marginar toda valoración ética de este monopolio. Es un juicio de hecho y no de valor lo que supone la expresión «con éxito».

De la misma manera se introduce, sin juicio de valor, el elemento de la legitimidad de la coacción física. Se trata de la aceptación interna de la coacción por parte de los dominados, de sus "motivos de justificación interior» (ibídem). He aquí, una vez más, el elemento subjetivo. La relación entre este elemento y el de "comunidad humana» es discutible. Lo específico de la forma nacional que alcanza el Estado en la época de los nacionalismos es precisamente que la legitimación del centro que monopoliza la coacción física se da en función de la creencia socialmente difundida de que el centro de poder emana de la comunidad que habita en el territorio, de la nación.

La condición del "éxito» se refiere tanto a la coacción física como a la legitimidad; sin embargo, no están en el mismo nivel de importancia el éxito en los dos monopolios; si no hay un mínimo éxito en el de la coacción física difícilmente podremos hablar de Estado, pues no existe un único centro de poder. Si la falta del éxito se refiere a la legitimidad, nos encontramos con que el Estado sí existe, aunque no sea legítimo para algunos o para muchos. La falta de legitimidad puede referirse tanto al ejercicio del poder como al centro de poder mismo. En un caso es el gobierno o la persona que ocupe el centro quien es ilegítimo; en el otro es el centro el que lo es, $y$, por tanto, también lo es el propio Estado, como ocurre en el caso de los nacionalismos periféricos ${ }^{5}$.

\subsection{El proceso histórico de modernización}

Habermas (1978) nos mostró el nacimiento histórico de la posibilidad de pensar la sociedad de que, progresivamente, desde la esfera privada, una serie de grupos sociales piensen en lo público y conciban la sociedad como unidad social total objeto de la política; en la substitución de un orden absoluto, cuya esfera pública era la corte, por un orden burgués en donde la sociedad se concibe a si misma y critica la política del poder.

La esfera pública se convierte en algo complejo, formado por la esfera pública del poder político y por la esfera pública generada desde la sociedad civil, desde la esfera privada. Fueron

4 Por ello es tan sorprendente, por lo menos, la libertad de posesión de armas de fuego existente en Estados Unidos.

5 Esta diferencia se muestra muy claramente en el caso español, con la muerte de Franco. Para algunos el franquismo había sido ilegítimo en función de la usurpación ilegítima del centro legítimo de poder, mientras que para otros el centro mismo de poder era ilegítimo, por no corresponder a la comunidad nacional de la que se sentian miembros. 
generándose una serie de mecanismos sociales a través de los cuales ciertos sectores sociales fueron pensando la sociedad como destinataria de la política. Después, progresivamente, se iría invirtiendo el orden jerárquico de las dos esferas, hasta alcanzar la idea de que el poder reside en el pueblo: la soberanía nacional. Siguiendo con la teoría de la diferenciación, la totalización de la sociedad es una operación política. Cuando se piensa la sociedad como totalidad que es el objeto de la política, se está pensando en que la esfera política representa a la sociedad. Voegelin tenía razón: es la política la que genera esa totalidad que los sociólogos hemos llamado sociedad. Y, para el caso concreto de los países europeos occidentales, Nisbet también la tenía: «la nación es la descendencia (offspring) del Estado» (1973: 164).

Habermas describe muy bien cómo surgen, desde la esfera privada, esos mecanismos de relación entre representantes y representados. Primero vinieron los salones - frecuentemente gestionados por mujeres de la alta aristocracia a los que acudian ciertos sectores cultos de esa clase social y de la nueva clase burguesa-, en los que se fue dando el salto desde la crítica literaria a la crítica política. Después vinieron los cafés, que cumplieron un parecido tránsito de lo literario a lo político ${ }^{6}$. Sin olvidarse de las sociedades secretas, más tarde vendrían la prensa periódica y, por fin, los partidos políticos, como canales sociales centrales de esta comunicación (Habermas, 1978: capítulo 2).

En la época de su formación, la mayoría de los Estados europeos occidentales con el establecimiento del territorio y del correspondiente poder central, ambos ya definitivos, contenían en el interior de su territorio poblaciones diversas, con culturas e, incluso, con lenguas diferentes. Durante la modernización se afianza - a través de los mecanismos puestos en marcha por la diferenciación frente al Estado de una sociedad civil que se va convirtiendo el objetivo de la política - una forma nueva de relación entre el poder político y la población que habita el territorio: la legitimación democrática nacional. El Estado dirigirá el proyecto moderno de homogeneización cultural de la población de diferentes grupos étnicos que habita dentro del territorio. El sistema nacional de educación, la red nacional de comunicaciones, el servicio militar obligatorio, serían, entre otros, mecanismos importantes de extensión de una cultura, una lengua y una conciencia nacionales?.

Sobre la dimensión subjetiva del Estado, su legitimidad, por Weber (1969: 1057), sabemos que no es una característica intrínseca de un poder, que puede existir sin legitimidad. Legitimidad es la disposición psicológica generalizada de aceptación de ese poder por parte de la población: consiste en «motivos de justificación interior», basados a lo largo del tiempo en la tradición, la religión o en la capacidad de un líder (carisma) para alzarse como legítimo frente a un poder visto como injusto. Cuando se formaron definitivamente los elementos objetivos del Estado, la religión fue un elemento imprescindible para legitimar un poder que se extendía a poblaciones con tradiciones y culturas religiosas diferentes. La homogeneización religiosa se hizo así relevante y por ello la aplicación en un momento histórico del principio cuius

6 Aunque este nuevo mecanismo social reflexivo excluyó sistemáticamente a las mujeres. Se puede recordar en este punto los grandes cafés de las grandes urbes europeas con sus tertulias exclusivamente masculinas y los panfletos contra esa droga maldita importada de América lanzados por las feministas de esas mismas ciudades.

7 Una forma normal de difusión de la lengua nacional fue la de elevar una de las lenguas propias de los diferentes grupos étnicos que habitaban el territorio del Estado a lengua nacional, para difundirla entre la población total a través del sistema nacional de educación y alfabetizar a quienes no habían alcanzado ese dominio de la lengua; en muchos casos los individuos pasaban de ser analfabetos en su propia lengua a estar alfabetizados en la lengua nacional, lo que significaba una clara mejora para acceder al mercado de trabajo. 
regio eius religio fue fundamental. Pero ya en la época de la modernización la cuestión de la legitimidad está cambiando radicalmente: el origen del poder sobre los hombres no reside en Dios ni en el rey como su representante, sino en los hombres mismos, en el pueblo, en la nación. Las monarquías debieron ser derrocadas o, al menos, sometidas al control político de la nación; y las Iglesias debieron ser apartadas de la esfera política pues su monopolio de la verdad y de la valoración ética del mundo debía ser sustituido por la voluntad popular.

Las ciencias, entre ellas la sociología, y las artes fueron fuerza creadora de esa cultura y esa identidad colectiva nueva, y el sistema educativo, sobre todo el público-nacional, fue el gran mecanismo difusor. Los historiadores hicieron el trabajo de contar la historia de la construcción del Estado como historia de la Nación, como si esta fuera anterior y hubiera conseguido, por fin, en ese momento de la historia, dotarse de una estructura política diferenciada y a su medida.

\subsection{El papel de la historia}

La historia "se convirtió en una disciplina privilegiada en el siglo XIX para dar un sentido de unidad subyacente en épocas pasadas que apuntaba a la unificación nacional y la identidad en el telos de la acción colectiva: Michelet para Francia, Prescott para los Estados Unidos, Macaulay para Gran Bretaña, Treitschke para Alemania son figuras representativas de los historiadores nacionalistas en este contexto. Y junto con la "historia", otros aspectos de la cultura estuvieron involucrados en la consolidación del Estado-nación, por ejemplo, la poesía y la música (esta última en forma de himnos patrióticos, himnos nacionales y, por supuesto, óperas, como las escritas por Verdi y Wagner)" (Tiryakian, 1989: 148).

"Las naciones modernas han sido construidas de manera diferente a como lo cuentan sus historias oficiales. Sus origenes no se pierden en la noche de los tiempos, en esas edades oscuras que describen los primeros capitulos de las historias nacionales. La lenta constitución de territorios al azar de conquistas y de alianzas no es tampoco la génesis de las naciones: no es más que la historia tumultuosa de principados o de reinos. El verdadero nacimiento de una nación es el momento en que un puñado de individuos declara que ella existe y comienza a intentar probarlo. Los primeros ejemplos no son anteriores al siglo xvIII. No hay nación en el sentido moderno, es decir político, antes de esa fecha. La idea, de hecho, se inscribe en una revolución ideológica. La nación es concebida como una comunidad amplia, unida por lazos que no son ni la sujeción a un mismo soberano ni la pertenencia a una misma religión (...). Todo el proceso de formación de la identidad ha consistido en determinar el patrimonio de cada nación, en difundir el culto.» (Thiesse, 1999: 11-12)

Nietzsche conocía bien el papel de la Historia en la construcción de mitos; para él, la finalidad del conocer residia en la destrucción de esos mitos de los que se alimenta el hombre. Por ello, como dice Foucault, para Nietzsche "la historia "efectiva" se distingue de la de los historiadores en que no se apoya sobre ninguna constancia. (...) Todo aquello a lo que uno se apega para volverse hacia la historia y captarla en su totalidad, todo lo que permite retratarla como un paciente movimiento continuo - todo esto se trata de destrozarlo sistemáticamente-. (...) La historia será efectiva» es decir, destructora de mitos, "en la medida que introduzca lo discontinuo en nuestro mismo ser. (...) Lo que se encuentra al comienzo histórico de las cosas no es la identidad aún preservada de su origen, es la discordia de las otras cosas, es el disparate» (1992: 19, 20 y 10). Sin embargo, la historia nacional suele ser enseñada como Historia Sagrada, sacralizada, para impedir así la manipulación mental de nues- 
tros orígenes, que son arbitrarios o, como diría Nietzsche, son la discordia de las otras cosas, el disparate, el conflicto... La función legitimadora de la Historia consiste en afirmar la existencia desde el origen de lo que no es sino un producto, un resultado histórico; según esto, la Nación existiría desde el origen y en la modernidad democrática lograría darse a sí misma una estructura política representativa. Pero la nación es un producto histórico moderno. Del mayor o menor éxito en la difusión de esta idea de Nación dependerá el surgimiento o resurgimiento de nacionalismos periféricos, de colectivos que lancen la idea de otra Nación contra aquel Estado. Por estas razones, Nisbet afirmaba con rotundidad que «la nación es la descendencia del Estado»" (1973: 164); y este Estado puede existir o ser mero proyecto.

Este es el modelo ideal de Estado que se intenta construir en Europa occidental en la modernización: el Estado nacional democrático. La nación no existía, el Estado tuvo que construirla a través de mecanismos sociales varios: el sistema nacional de educación, que transformaba a los analfabetos en su lengua materna en alfabetos en lengua nacional, las guerras con el exterior, el servicio militar obligatorio...

El proceso histórico de construcción de este tipo de Estados no puede entenderse sin un proceso de industrialización que suponía la aplicación sistematizada de las consecuciones científicas y tecnológicas a la producción fabril, e implicaba el nacimiento de un nuevo sector social que fue la primera clase nacional de la historia (Marx y Engels, 1972: 71), clase que necesitaba para el desarrollo de sus actividades económicas la conjunción territorial del Estado y del mercado protegido por ese Estado.

\subsection{Los países rezagados}

Tiryakian (1989: 147-152) describe una serie de sucesivas oleadas de nacionalismo en la historia humana (ver cuadro 1). La primera de ellas es la correspondiente a la entrada en escena de los Estados-nación en Europa y en Estados Unidos en la segunda mitad del siglo xVIII y a comienzos del XIX; esta oleada incluye también a ciertas regiones periféricas europeas que tenían un importante grado de identidad cultural, pero carecían de autonomía o unidad política (Grecia, Italia, Alemania). Todos estos casos son los que han constituido la referencia fundamental para los investigadores de los casos posteriores de transición política en diferentes partes del planeta. Este modelo de referencia es absolutamente necesario para los nacionalismos étnicos periféricos que se desarrollan a finales de los sesenta y en los setenta del siglo $x x$ en contra de los Estados-nación occidentales ya establecidos, y que constituyen la tercera ola; es lógico, pues al ser nacionalismos de secesión de un Estado democrático ya constituido, cada uno de ellos es reflejo especular del nacionalismo que originó la transformación del Estado en un Estado-nación. De manera que los elementos del modelo fundamental siguen siendo los del nacionalismo periférico, aunque la dimensión histórica nos permite observar las diferencias y contradicciones entre el nacionalismo con Estado y el del que no lo ha conseguido, pues el éxito de uno constituye el fracaso del otro.

8 Las excepciones europeas (Grecia, Italia, Alemania) en las que la idea nacional es anterior a la unificación territorial no hubieran sido seguramente posibles sin un contexto europeo en el que imperaban los procesos de construcción de la idea de que la población del Estado era una nación y de difusión por ese Estado de la idea y el sentimiento nacionales por todo el territorio. El caso de Turquía es diferente, por las fuertes tensiones, todavía vigentes, entre quienes proponen diferentes vías de estabilización política. 


\section{Cuadro 1}

Oleadas de nacionalismos, según Tiryakian (1989: 147-152)

«El orden social moderno que se apoya en una economía industrial, una interpretación científico-racional del mundo y una política burocratizada personificada en el estado-nación hizo su aparición en Europa Occidental en la segunda mitad del siglo XVIII. Acompañando a la llegada de este orden social aparecieron varias manifestaciones de nacionalismo. Francia (...) con la visión jacobina de la nación "una e indivisible" (...) el nacionalismo americano (...) como la consciencia de que las "colonias" habían evolucionado hacia realidades socio-políticas diferentes de la realidad de Gran Bretaña. (...) la primera nación en industrializarse, Inglaterra misma (...).

Los casos de Grecia, Italia y Alemania son paradigmáticos ya que el nacionalismo supuso el derrocamiento de la dominación extranjera, y la elaboración de una identidad nacional basada en el despertar de una trayectoria política común y la construcción de identidades nacionales. (...).

El ejemplo americano (...) fue seguido por las guerras de independencia de los territorios hispanos en el Nuevo Mundo. El resultado fue la "Balcanización" del antiguo imperio español (...). En Europa, el nacionalismo como demanda de autonomía del control extranjero se convirtió en la segunda mitad del siglo XIX en un agudo problema para el Imperio Austro-Húngaro, especialmente sentido por las minorías eslavas; este fue igualmente el caso (...) de las minorías no turcas, árabes y cristianas en la misma medida, en la decadencia del Imperio Otomano.

(...) el resultado de la primera guerra mundial, el tratado de Versalles, y el establecimiento de la Liga de Naciones, marcaron el final de la primera oleada (...) El resultado (...) fue engañoso: exteriormente, varios estados-nación nuevos aparecieron en la Europa Central y del Este, pero los Aliados que administraban la paz bloquearon otras demandas de autonomía, especialmente las de sus colonias y protectorados en Asia, Oriente Medio y África.

La segunda oleada importante (...) empezó a desarrollarse en el periodo de entreguerras en las mismas unidades que se habian incorporado a los nuevos imperios. Cuando las poblaciones se dieron cuenta de que la promesa de inclusión en una nueva sociedad mayor era un mito y de que solamente en el mejor de los casos una pequeña porción de la población indígena podía participar en los procesos de toma de decisiones como ciudadanos de pleno derecho, entonces tuvo lugar el desencanto (...) y finalmente la movilización política contra la "metrópoli" extranjera (...).

Esta ola de nacionalismo tuvo varias vertientes. En el primer período (desde la década de 1930 a la de 1950) algunos aspectos del nacionalismo en el Tercer Mundo tomaron la forma de buscar la reunificación de pueblos que habían estado fragmentados políticamente por la demarcación imperial (ver, por ejemplo, Hodgin (1957) para casos en África) (...).

Una vertiente importante de esta segunda ola (...) ha sido la formación de un estado-nación políticamente autónomo a partir de una unidad administrativa (o colonia) del anterior imperio, (...) después de violentas luchas y de guerras internas (Indochina, Argelia, Angola) (...) en Asia, África y el Oriente Medio, una cantidad significativa (...) tuvo lugar sin recurrir a la lucha armada. (...) También hay que incluir aquí (...) el nacionalismo comunista (...) en el Tercer Mundo, la movilización del apoyo popular a la revolución socialista ha tomado la forma de buscar el desalojo del control extranjero y el desarrollo nacional del país como una totalidad. La segunda oleada (...) alcanzó su momento álgido en la década de 1960 (...) África del Sur (...) es la escena de un poderoso nacionalismo urbano negro que ha forjado la unidad de grupos étnicos de orígenes diferentes (...) hay un vigoroso nacionalismo de estados-nación en el Este de Asia que han pasado a ser actores internacionales en la economía del globo (...).

Una tercera ola de nacionalismo (...) se desarrolló a finales de los años sesenta y en la década de los setenta; se trata del grupo de movimientos nacionalistas que surgió dentro y en contra de estados-nación occidentales establecidos (...) Canadá, Francia, Gran Bretaña y España.»

Elaboración propia. 
Pero las otras transiciones, a lo largo y ancho del mundo responden, a formas generalmente diferentes, aunque el modelo de referencia sigue siendo central para el investigador, porque la falta de adecuación de ese modelo pondrá de relieve las nuevas características del caso sometido a análisis. Una finalidad fundamental de todo modelo teórico es la de ser destruido al contrastarlo con una realidad concreta (Badiou, 1969: 17). Por esta razón, mi conclusión será extraer para el investigador de los nuevos casos una estrategia pragmática que contiene una fuerte dosis de precaución.

Un trabajo sobre la transición en el sur de Europa y en América Latina que ha tenido mucha repercusión académica fue dirigido por O'Donnell y Schmitter (1986); sus conclusiones pasaron a ser una referencia canónica sobre el tema. En la parte referente al sur de Europa, Schmitter expone (cuadro 2) cómo han sido caracterizadas las transiciones de Italia, Grecia, Portugal, España y Turquía.

\title{
Cuadro 2
}

Las transiciones del sur europeo, según Schmitter (1986: 3-4)

\begin{abstract}
"Los países del borde norte del Mediterráneo han sido durante mucho tiempo los "hijastros" del estudio de la política y la sociedad de Europa occidental. Con la notable excepción de Italia (y solo desde su democratización después de la Segunda Guerra Mundial), se los ha colocado de manera rutinaria fuera de la corriente principal de investigación y generalización sobre los desarrollos políticos en esa parte del mundo. Los eruditos evitaban estudiarlos. Los libros de texto simplemente ignoraron su existencia. Los sistemas de clasificación les asignaron el estado de "excepciones", o simplemente los colocaron en la ignominiosa categoría de "otros" (...).
\end{abstract}

Creció la convicción de que de alguna manera no pertenecen a Europa Occidental. España y Portugal fueron colocados al otro lado de los Pirineos, es decir "en África". Grecia, cuando cayó bajo el despotismo de las coroneles, llegó a ser balcánica. Turquía, a pesar de sus esfuerzos por secularizarse y modernizarse a la occidental, fue exilada al Oriente Medio.

Los capítulos de este libro que tratan de la desaparición del gobierno autoritario en el sur de Europa y de la lucha para establecer la democracia política, implícitamente arguyen que estos países - Italia hace algún tiempo; Portugal, España y Grecia más recientemente; Turquía más ambiguamente- han alcanzado, y puede esperase que permanezcan en él, el rango de variación institucional y los modelos de conflicto político propios de Europa Occidental como totalidad.

Quizás más que cualquier otra cosa, esta conclusión marca el contraste más fuerte con los casos latinoamericanos que han sido también parte de este proyecto. (En general, los autores de estos estudios sobre el sur europeo, exceptuando el caso de Turquía) tienen relativa confianza en que una transición del régimen ha ocurrido en el sur de Europa y en que, a pesar de las incertidumbres persistentes y de los problemas no resueltos, las democracias políticas que han surgido para reemplazar al gobierno autoritario tienen una posibilidad razonable de sobrevivir. Los colegas latinoamericanos no muestran ese optimismo. Algunos dudan de que haya comenzado siquiera una transición; otros no están seguros de qué modo de dominación política reemplazará a las autocracias extintas; $y$ otros dudan en suponer que las nuevas democracias sucesoras podrán consolidarse en un futuro próximo.»

Elaboración propia.

La edición posterior de los resultados del proyecto fue prologada por Arnson y Lowenthal (2013), quienes prolongaron el sentido del historial de las transiciones estudiadas en el proyecto (cuadro 3). 


\section{Cuadro 3}

Las transiciones actuales y futuras, según Arnson y Lowenthal (2013: XII y XIII)

"La mayoría de las transiciones desde el gobierno autoritario en los años 1970s y 1980s, y unas pocas en los 1990s, tuvieron lugar en países que habian tenido al menos alguna experiencia previa de gobierno democrático constitucional. En algunos casos, instituciones democráticas todavía tenían presencia. Las transiciones de hoy y las de mañana desde formas autoritarias de gobierno que están ocurriendo o que lo harán se darán en países con pequeña o nula experiencia previa.»

Elaboración propia.

En el año de esta segunda edición, 2013, la Guerra Fría había terminado, el Telón de Acero que protegía el Imperio soviético se había desmoronado, el mundo islámico se había escindido en movimientos moderados y otros fundamentalistas y la globalización de la economía estaba ya succionando la capacidad de los Estados plenamente constituidos para regular la vida social. Pero, además, por otra parte, la Unión Europea, como referencia central en los procesos de transición política, estaba perdiendo peso; por un lado, los países asiáticos y los africanos estaban en una relación probablemente menos intensa en términos culturales con el mundo europeo $y$, por otra, el peso de la histórica colonización europea de esos países era muy fuerte; la democratización ha podido llegar a ser concebida como europeización u occidentalización.

\subsection{Un último apunte sobre los países europeos que se modernizaron}

Tilly (1990) nos mostró las dos leyes que definieron el "sistema europeo de Estados» hasta 1990: cada Estado, culturalmente, fue progresivamente más homogéneo en su interior, y cada vez los Estados fueron más diferentes entre ellos. En nuestros días las dos leyes se han quebrado en el nuevo escenario global. La progresiva homogeneidad cultural interna de cada Estado desaparece por las migraciones (económicas y políticas) internacionales que comenzaron tras las guerras mundiales - y que en nuestros dias la Unión Europea ni siquiera ha comenzado a regular - por el hecho de que los dos modelos que funcionaron en estos países para integrar la población inmigrante mostraron su ineptitud, a raíz de la crisis del petróleo que comenzó en 1973; por un lado, las condiciones de entrada de nuevos inmigrantes se endurecieron brutalmente, $y$, por el otro, la segunda generación de origen inmigrante llegaba al poco tiempo del comienzo de la crisis a intentar comenzar su integración definitiva concurriendo al maltrecho mercado de trabajo con escaso éxito; las reacciones de esta segunda generación han sido muy fuertes.

Y la ley de la progresiva heterogeneidad entre los Estados también se ha quebrado porque todos los países son hoy pluriculturales en donde la población de origen inmigrante ha recreado ${ }^{9}$ su cultura originaria en Europa (Wieviorka, 1998), y, por otra parte, una de las características de la globalización consiste en la progresiva occidentalización cultural del mundo, también llamada macdonalziación (Ritzer, 1992 y 1996).

9 En Kivisto (2014: 61-87) podemos ver las formas de re-creación de la organización eclesial por la población inmigrante, muchas veces muy cercanas a las formas de organización de las confesiones autóctonas. 
Los países europeos están atravesando una situación de crisis importante: la interpenetración entre sociedad y política no es una realidad que esté garantizada, es preciso mantener la relación de comunicación entre la esfera política del Estado y la esfera civil de la sociedad, que alcanzaba a tener una dimensión pública, política: en ello consiste el sistema democrático representativo de gobierno. Los partidos políticos eran vehículo de transmisión a la esfera política de las necesidades y problemas de la población. La relación parece haberse invertido: los partidos y la clase política pretenden comunicar a la ciudadanía cuales son las necesidades de la población, se han ido autonomizando de la sociedad civil, de los ciudadanos. En la tabla 1 puede observarse el bajo grado de confianza que los europeos en general y, más bajo aún, los españoles ${ }^{10}$ tienen en sus instituciones políticas.

\section{Tabla 1}

Identidad europea y confianza de los españoles y de los europeos en las instituciones propias y europeas

\begin{tabular}{|c|c|c|c|c|c|c|c|c|c|}
\hline A & & $\begin{array}{l}\text { Se siente } \\
\text { ciudadano } \\
\text { de la U.E. }\end{array}$ & $\begin{array}{c}\text { Se } \\
\text { siente } \\
\text { unido } \\
\text { a la U.E. }\end{array}$ & $\begin{array}{c}\text { Confia en } \\
\text { instituciones } \\
\text { de la U.E. }\end{array}$ & $\begin{array}{l}\text { Parlamento } \\
\text { europeo tiene en } \\
\text { consideración } \\
\text { problemas } \\
\text { ciudadanos } \\
\text { europeos }\end{array}$ & B & $\begin{array}{l}\text { En la } \\
\text { U.E. }\end{array}$ & $\begin{array}{c}\text { En } \\
\text { Parlamento } \\
\text { nacional }\end{array}$ & $\begin{array}{l}\text { En } \\
\text { Gobierno } \\
\text { nacional }\end{array}$ \\
\hline \multirow{2}{*}{ 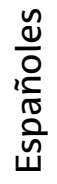 } & Sí & $75 \%$ & $69 \%$ & $38 \%$ & $27 \%$ & Confian & $16 \%$ & $8 \%$ & $10 \%$ \\
\hline & No & $24 \%$ & $31 \%$ & $59 \%$ & $69 \%$ & No confian & $79 \%$ & $89 \%$ & $89 \%$ \\
\hline \multirow{2}{*}{ 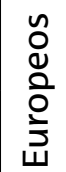 } & Sí & $63 \%$ & $62 \%$ & $43 \%$ & $38 \%$ & Confían & $31 \%$ & $28 \%$ & $27 \%$ \\
\hline & No & $35 \%$ & $36 \%$ & $52 \%$ & $54 \%$ & No confian & $56 \%$ & $65 \%$ & $68 \%$ \\
\hline
\end{tabular}

Fuente: Pérez-Agote, 2016b: 107.

Si nos fijamos en los ciudadanos españoles, podemos extraer interesantes conclusiones: a) el sentimiento de pertenencia a Europa es más fuerte que la confianza en sus instituciones; b) su confianza en estas es menor que la media europea; c) aún así tienen mucha mayor confianza en las instituciones europeas que en las españolas; d) la confianza en las instituciones española es absolutamente pequeña. Si miramos el Barómetro del Centro de Investigaciones Sociológicas de Julio de $2014^{11}$, vemos cuáles son los problemas que preocupan a los españoles: tras el paro ${ }^{12}$, que lo es para el $54,8 \%$ de ellos, vienen la corrupción $(15,7 \%)$ y, en tercer lugar, «los/as políticos/as en general, los partidos y la política» (10\%). En los últimos años, la

10 He escogido en especial España por el fuerte grado de crisis económica alcanzado, porque en este país ha surgido un movimiento de protesta contra dicha autonomización y, también, porque tuve la gran suerte de observar los momentos fundacionales de ese movimiento junto al profesor Tiryakian en la madrileña Puerta del Sol.

11 Disponible en: http://www.cis.es/cis/opencm/ES/1_encuestas/estudios/ver.jsp?estudio=14099

12 En 2007 la tasa de paro que estaba en torno al 8\% comenzó a crecer rápidamente comenzó a crecer. En 2011 había casi triplicado. En septiembre de 2014, está en torno al $24 \%$ y para los menores de 25 años entorno al 54\%. Disponible en: http://www.datosmacro.com/paro/espana 
corrupción ha venido alcanzando cotas cada vez mayores de preocupación pública; los políticos y la política han ido pasando de ser parte de la solución a ser problema.

Esta frustrante situación fue atravesada por la epidemia global de movilización política juvenil que se había iniciado con la Primavera Árabe ${ }^{13}$. A pesar de que las situaciones políticas y económicas de partida fueran muy diferentes se trataba de una epidemia global, con características comunes: el grupo motor de la movilización fue la juventud instruida con problemas para incorporarse al mercado de trabajo; se luchaba siempre por un sistema político democrático que se ocupara de los problemas reales de la población, independientemente del concreto régimen político del país, lo que hace pensar en una cierta extensión a escala global de la idea de democracia; se ocupaban pacíficamente espacios sociales urbanos socialmente centrales y significativos; se utilizaban masivamente las nuevas tecnologías de la comunicación; se procuraba una democracia directa, impidiendo activamente la génesis de un centro.

En España, en febrero de 2011, bajo la denominación genérica de Democracia Real Ya, nació una plataforma en las redes sociales que empezaba a aglutinar toda una serie de movimientos y asociaciones, principalmente juveniles. Esta plataforma logró convocar manifestaciones en 40 ciudades que se celebraron el 15 de mayo ${ }^{14}$. Este movimiento, el $15 \mathrm{M}$, abrió un intenso proceso de dinamización de la vida social y política, siendo algunos de sus aspectos relevantes desde el punto de vista de la diferenciación social. El leitmotiv del movimiento 15M fue la crisis de la relación entre la esfera política y la sociedad civil. El movimiento tenía una doble vertiente: elaborar una nueva cultura política, una nueva forma de estar políticamente en la sociedad civil y modificar en profundidad la actividad de la esfera política para que su objetivo fuera solucionar los problemas de la población. Se trataba de producir efectos en las dos esferas. En la esfera política, que se había independizado de la esfera de la sociedad civil. Y en esta esfera, que se había habituado al consumo (posición pasiva) de la política a través de los medios de comunicación social. En definitiva, toda una serie de personas, redes sociales, plataformas, reivindicaciones, acciones que intentaban restablecer una relación de la política con la sociedad. El espectro organizativo interno fue muy amplio, yendo desde ciertas movilizaciones sociales puramente locales, que se amparaban simbólicamente en el $15 \mathrm{M}$ como receptáculo simbólico, hasta la formación de un partido político (Podemos) ${ }^{15}$; entre esos extremos, se formaron otro tipo de organizaciones que se fortalecieron al entrar en la Plataforma de Afectados por la Hipoteca (PAH), o de proto-partidos (Partido X) que trataron de patrocinar nuevos mecanismos para posibilitar la participación ciudadana en la política convencional. Un movimiento de estas características mantiene periodos intermitentes de visibilidad y de invisibilidad. La pregunta fundamental es por la posible relativa unidad simbólica del movimiento y por el mantenimiento de algo así como un caldo de cultivo que permita esa relativa imagen de unidad simbólica sin necesitar una mínima organización que la sostenga en el tiempo ${ }^{16}$.

13 El 17 de diciembre de 2010 un joven tunecino de 26 años se inmolaba a lo bonzo. Fue el inicio simbólico de esta epidemia.

14 Disponible en: http://sociedad.elpais.com/sociedad/2011/05/11/actualidad/1305064806_850215.html

15 Organización crecientemente centralizada en aras de la eficacia, pero intentando conservar la referencia a una nueva forma de hacer política, en comunicación directa y constante con la sociedad civil, sin formar parte de la casta separada.

16 En un trabajo anterior (Pérez-Agote, 2016a) planteaba el difícil problema de la articulación entre el movimiento genérico e intermitente y el partido surgido de ese movimiento, Podemos, que actualmente participa en el Gobierno español presidido por el Partido Socialista (PSOE). 


\section{HACIA UNA ESTRATEGIA DE APERTURA DE LA TEORÍA DE LA TRANSICIÓN PARA EL ANÁLISIS DE OTROS CASOS}

La noción de modernidad presenta dificultades. La primera es una dificultad general derivada de que surge a partir de una serie de países concretos de una zona concreta del planeta, que en unos momentos históricos estaban relacionados entre sí, como consecuencia de procesos bélicos, paces y tratados, herencias y matrimonios de príncipes y reyes, y procesos parciales de una religión que había sido común; y todos estos aspectos y relaciones van a converger en un sistema europeo de Estados, sistema cuya primera concreción la podemos ver en la Paz de Westfalia (1648). Esto nos hace pensar en que el proceso de los países rezagados de la zona (Grecia, Italia, Alemania ${ }^{17}$ ) es un proceso similar al de los otros países europeos occidentales, aunque el orden de aparición de las dos instituciones fundamentales (Estado y nación) que están implicadas en una relación de causalidad sea el inverso; la razón es que en los tres casos de retraso, aunque la nación preceda al Estado, la idea de Estado preexiste y forma parte de la propia idea de nación. No es posible pensar en la nación de forma concreta sin referencia al Estado correspondiente, sea realidad presente o previsión para el futuro.

Esta forma concreta de interconexión entre las diferentes realidades sociales de la zona no tiene por qué estar presente en otros casos. Otra dificultad que presenta la noción de modernidad derivada radica en que su significación penetra en varios aspectos de la realidad social: en la dimensión política, en la económica, en la cultural, en la religiosa, en la científicotécnica. La sociología, como teoría de la modernización que fue desde sus inicios del siglo XIX hasta los años setenta del xx, establecía una única vía posible para alcanzar la modernidad, que era la seguida por los países occidentales; y ahora, en la actualidad, admite la pluralidad de vías para alcanzarla (Eisenstadt, 2000, 2001); pero con ello, la noción de modernidad va perdiendo su referencia a la pluralidad de esferas implicadas en aquel proceso; está disminuyendo la complejidad del sentido y, con ello, la noción de modernidad queda reducida progresivamente a un alto nivel de desarrollo económico; es particularmente relevante la omisión de referencias políticas al tipo de Estado al que da lugar cada proceso concreto, así como el proceso en Europa occidental supuso la puesta en funcionamiento, en cada país, de un Estado nacional democrático representativo.

Por esta razón, una teoría de la transición ha de llevar al investigador a partir, en su análisis de un caso concreto, de una doble vía de reflexión y análisis. Por un lado, es necesario que se formule la pregunta sobre cuál era el objetivo final del proyecto político - político porque fue el Estado históricamente ya constituido el principal agente ejecutor de ese proyecto- en el caso de los países de Europa occidental. A mi modo de ver, las preguntas en concreto son sobre qué problema se pretendía resolver con la transformación del Estado en Estado nacional democrático representativo, sobre qué sector social impulsaba el proceso y sobre qué mecanismos se pusieron en marcha para llevarlo a cabo; la respuesta es compleja, pero sin duda va en la dirección siguiente: la nueva burguesía industrial - primera clase nacional de la historia (Marx y Engels, 1972: 71) - se propone la transformación del Estado en el sentido de que el objeto de la política - que es la actividad del Estado- sea la sociedad, la población que se encuentra dentro del territorio controlado por ese Estado, que no será ya la administración del patrimonio real. Este es el problema central que el proyecto de modernización pretende

17 Cf.: nota 7 . 
resolver por medio de la transformación del Estado en un Estado nacional democrático representativo; la finalidad del proyecto, en el trasfondo, es, en primer lugar, la conexión entre la actividad del poder político y la población habitante del territorio; $y$, en segundo lugar, dar la preminencia a la población, concebida ya como sociedad, sobre el Estado y la clase política. y, por el otro lado, el investigador tendrá que analizar si el objetivo del proceso emprendido en el país que fuere es el de llevar al Estado, al poder político a tener como objetivo de su actividad la población que habita el territorio y a transformar las instituciones para dirigirse hacia este objetivo; se deberá analizar el papel del Estado y de las instituciones en el desarrollo de la actividad política; será relevante, además, desvelar las posibles formas de control social de esas instituciones y de ese Estado por la población; también deberá describir los mecanismos que se están desarrollando para la progresiva aceptación por la población de la nueva institucionalización política; $y$, por último, el investigador deberá dirigir su atención a describir los sectores sociales que son los agentes instigadores del proyecto.

\section{UN EJEMPLO, CONTEMPORÁNEO Y COMPLEJO, DE ANÁLISIS CONCRETO DE UNA ZONA EN PLENO PROCESO DE TRANSICIÓN: ÁFRICA DEL OESTE DE ANTERIOR COLONIZACIÓN FRANCESA}

Conociendo la fuerte atención que dedica esta revista a las sociedades latinoamericanas, y dada la mayor similitud de los problemas de estas con los de Europa occidental por su pertenencia a la primera oleada de nacionalismo, y sus esfuerzos históricos por conseguir consolidar democracias nacionales representativas, he escogido detenerme en casos del África subsahariana por ser una realidad menos cercana en términos sociológicos y políticos, $y$, también, también por ser menos frecuente en nuestro ámbito la publicación de trabajos sobre las enormes dificultades que arrastran las sociedades de esa zona del planeta. Y otra razón, más pragmática, ha sido tener la suerte de poder vivir una relación fuerte con ese continente ${ }^{18}$.

Una obra recientemente publicada por Holder y Dozon (2018), sobre los países de África del Oeste salidos de la colonización francesa (Burkina Faso, Gabon, Mali, Niger y Senegal), es resultado de un proyecto de investigación titulado Espaces publics religieux. États, sociétés civiles et islam en Afrique de l'Ouest. Este trabajo entra de lleno en el ámbito de problemas que estoy tratando de ordenar teóricamente, por lo que constituye un buen ejemplo de las diferentes formas de búsqueda de una forma de ordenación de un Estado a los intereses y necesidades de una población; esta búsqueda da lugar a la consideración de la población como sociedad que se está concibiendo y, por ello mismo, constituyendo como tal sociedad.

18 Mi primer contacto con la zona subsahariana de África vino porque el Presidente de la AISLF (Association Internationale des Sociologues de Langue Française), Renaud Sainsaulieu, me invitó a participar un coloquio titulado Démocratie, culture et développement. Contextes et enjeux sociologiques en Afrique Aujourd'hui celebrado en Bénin, en la ciudad de Cotonou (23-26 de enero de 1995), con la pragmática decisión política de caminar hacia la constitución de un Grupo de Trabajo titulado "Sociétés Africaines» que daría lugar después al actual Comité de Investigación 37, Afriques en mutation, al que sigo perteneciendo. En el Boletín n. ${ }^{\circ} 11$ de la Asociación publiqué un pequeño artículo cuyo título era «Touché!». Relataba la conmoción personal y sociológicas que me produjo la visita. Actualmente colaboro en temas de investigación con la inmigración senegalesa de Madrid. 
La noción teórica que define la obra es la de espacio público religioso, que permite analizar las reconfiguraciones del islam en esta zona africana hacia fines del pasado siglo $x x$, porque la ola de Conferencias Nacionales africanas había dado lugar a una sociedad civil, liberalizando el derecho de asociación. En esta atmósfera pre-democrática, las asociaciones musulmanas nacieron y desarrollaron una forma práctica, africana, de islam; y esta nueva forma se definirá como no violenta y respetuosa con las instituciones. Pero los préstamos del Banco Mundial a los países de la zona estuvieron condicionados a los programas de ajuste estructural del Fondo Monetario Internacional, con consecuencias muy negativas en términos de empleo, servicios públicos y costo de vida. Esto produjo el nacimiento de importantes proyectos de emancipación liderados por asociaciones musulmanas (Mali, Níger, Senegal) o por movimientos neopentecostales (Burkina Faso, Costa de Marfil). «En África, mientras el Estado opera resueltamente un traspaso de la razón política al ámbito económico, la sociedad realiza así un traspaso paralelo de esta misma razón a un ámbito religioso que no es consensual en absoluto» (Holder y Dozon, 2018: 28).

El trabajo de Dozon (2018) sobre Senegal (bajo la presidencia de Abdoulaye Wade, 20002012) parte de la idea de este país como "nación de hermandades» (nation confrérique), noción que incluye otras nociones: la de «acomodación» recíproca entre los colonizadores franceses y el mundo islámico senegalés, la de "contrato social senegalés» entre el poder estatal y el poder de la hermandad y el de la "coproducción». Desde la época colonial, las hermandades y el Estado han coproducido, y esta coproducción ha dado forma a la nación senegalesa. El poder político se había debilitado primero por las sequias y luego por los programas de ajuste estructural. En su desilusión política y su ilusión creada por la revolución iraní, una parte importante de los militantes de movimientos de tendencia marxista vino a fortalecer el mundo de las hermandades. El debilitamiento del Estado y la política fue compensado por el fortalecimiento político de las hermandades y especialmente la de los Mouridiyya, que apoyaron el poder de Wade.

A lo largo de los capítulos de esta obra, podemos ver una forma diferente de construir un espacio público desde el ámbito religioso islámico. Después de la independencia, momento de la determinación precisa de los elementos objetivos del Estado, se produce un proceso de democratización de ese Estado que da lugar a la creación de asociaciones religiosas; pero, a pesar de ello, la población ve la democratización como una forma de occidentalización. Las transformaciones estructurales impuestas por el Fondo Monetario Internacional y el Banco Mundial dejarán a los Estados con una capacidad muy limitada de incidencia en las áreas de educación, salud y ayuda humanitaria. Y estas funciones se irán realizando paulatinamente a través del mundo de las asociaciones religiosas, que en muchos casos se convierten en ONGs. Este papel cívico / político que juega el ámbito islámico contrasta con el modelo de Europa Occidental, en el que se produce un proceso de separación entre Iglesia y Estado y la emergencia de una esfera pública no religiosa que emerge desde la esfera privada. Tal vez habría sido muy relevante la discusión de si el término nación, que fue estratégicamente decisivo en el caso de los países europeos occidentales, no sea el más adecuado para denominar el conjunto asociativo religioso de esas nuevas "sociedades» que se están generando en el proceso. En el caso europeo, la nación, desde el punto de vista de la población que habita el territorio, es la idea de sociedad que en el proceso de modernización/democratización logra su institucionalización política en el Estado democrático, un Estado que, por lo mismo, está en comunicación con la nación; pero, paradójicamente, el sociólogo conoce que el Estado logra, a través de determinados mecanismos, difundir mayoritariamente la idea entre la población; en el 
caso más general, es el Estado el antecesor que genera la nación, que alcanza su sentido político asumiendo que la nación precede al Estado.

El trabajo de esta investigación nos muestra cómo la dimensión cívica y humanitaria del asociacionismo islámico adquiere un relieve que supera en ocasiones al de la dimensión religiosa. Además, es interesante pensar en las formas tan plásticas y flexibles que adopta esta religión. Por un lado, se trata de una religión de libro, lo que implica cierto grado de institucionalización, aunque es evidente que de este libro se derivan diferentes escuelas y tradiciones interpretativas. Pero, por otro lado, la autoridad religiosa en el mundo islámico está más dispersa que en el mundo católico, y esta cierta atomización institucional islámica conduce eventualmente a una mayor capacidad de adaptación a nuevos enclaves sociales. En estos países del islam africano, es claro que el islam es categóricamente no violento y respetuoso con las instituciones políticas, pero su flexibilidad permite que las asociaciones religiosas jueguen un papel político fundamental, en muchos casos llevando a cabo un trabajo cívico y humanitario que el Estado, económicamente débil, no puede desarrollar. Puede ser interesante preguntarse sobre la influencia de la cultura animista profunda en estas formas flexibles de adaptación de la religión; también sobre las relaciones de competencia, colaboración y legitimación entre el Estado y el mundo asociativo religioso; $y$, finalmente, sobre el papel que jugó el mundo religioso en el trance que, en los años 90, llevó a los países de esta región del planeta a considerar la institucionalización de una nación democrática.

Para terminar con esta propuesta de un modelo analítico de la transición, es preciso entender esta como el proceso de transformación de una realidad social, la población que habita un territorio controlado por un Estado, en lo que constituye una sociedad para los sociólogos; esto implica la transformación de un Estado existente, que adopta una forma de organización política destinada a resolver los problemas de esa población que, a su vez, se constituye en un espacio público que se comunica con el aparato del Estado y ejerce un control sobre este. Por lo tanto, un proceso de transición supone la existencia previa de un Estado.

Volviendo a la realidad africana retenida para el análisis, en todo momento se ha dado por supuesto la construcción del proceso de transformación de un Estado ya existente, heredado de la colonización, francesa en ese caso. Esta aceptación pragmática de las fronteras heredadas se planteó como mal menor desde los comienzos de los procesos de independencia política de los diversos países, e incluso, fue uno de los primeros problemas que tuvieron que afrontar estos países y fue prontamente tratado por las organizaciones que precedieron a la actual Unión Africana ${ }^{19}$.

La cultura política profundamente europea y occidental a la que pertenezco, y también moderna y democrática - por la que tuvimos que luchar quienes nos socializamos políticamente durante la dictadura - recela inmediatamente de esta modelación política del islam; en Europa occidental hemos tenido $-y$, en algunos casos, seguimos teniendo- que recelar de todas las presiones políticas que provienen de la esfera religiosa. Es preciso evitar los juicios de valor y tratar de indagar si una construcción política diferente tiende a solucionar el problema previo fundamental que el proceso europeo de modernización trató de resolver. Más

19 La primera de ellas fue la Unión de Estados Africanos (1958-1962), seguida de la Organización para la Unidad Africana (OUA, 1963-2002), y de la Unión Africana (UA, 2002-presente). Fue particularmente difícil el caso de la región de los Grandes Lagos, en el que se llegó a la propuesta de hacer coincidir el territorio con la composición étnica: sustituir Ruanda y Burundi por Hutulandia y Tutsilandia, dos Estados étnicamente homogéneos. Pronto se reconoció que generaba más problemas de los que resolvía. 
aún, Europa occidental fue parte bien activa en la configuración de la difícil situación social y política en la que se encuentran sus viejas colonias: una razón más para afirmar que la finalidad de un modelo en el análisis de otra realidad es mostrar tanto lo que se asemeja y como lo que se separa.

\section{REFERENCIAS}

Abercrombie, N., Hill, S., y Turner, B.S. (1984). Dictionary of Sociology. Hardmondsworth, London: Penguin.

Arnson, C.J., y Lowenthal, A.F. (2013). Foreword. En G. O'Donnell y P.C. Schmitter (Eds.), Transitions from authoritarian rule: Tentative conclusions about uncertain democracies (pp. vii-xiv). Baltimore: Johns Hopkins University Press.

Asad, T. (2003). Formations of the Secular: Christianity, Islam, Modernity. Stanford: Stanford University Press.

Badiou, A. (1969). Le concept de modèle. París: Maspéro.

Casanova, J. (2006). Secularization revisited: A reply to Talal Asad. En D. Scott y C. Hirschkind (Eds.), Powers of the Secular Modern: Talal Asad and His Interlocutors (pp. 12-30). Stanford: Stanford University Press.

Dozon, J.-P. (2018). Le Sénégal de Abdoulaye Wade: entre populisme et affirmation mouride. En G. Holder y J.-P. Dozon (Dirs.), Les politiques de l'islam en Afrique. Mémoires, réveils et populismes islamiques (pp. 203-214). París: Éditions Karthala.

Elias, N. (1982). La Sociedad Cortesana. Ciudad de México: Fondo de Cultura Económica.

Eisenstadt, S.N. (Ed.) (2000). Multiple modernities. New York\&London: Routledge.

Eisenstadt, S.N. (Ed.) (2001). Comparative civilizations and multiple modernities (Vol. 1). Leiden: Brill.

Foucault, M. (1992). Microfisica del Poder. Madrid: La Piqueta.

Habermas, J. (1978). L'espace public. Archéologie de la publicité comme dimension constitutive de la société burgeoise. París: Payot.

Holder, G., y Dozon, J.-P. (Dirs.) (2018). Les politiques de I'islam en Afrique. Mémoires, réveils et populismes islamiques. París: Éditions Karthala.

Kivisto, P. (2014). Religion and Immigration. Migrant Faiths in North America and Western Europe. Cambridge: Polity Press.

Luhmann, N. (1990). The paradox of system differentiation and the evolution of society. En J.C. Alexander y P. Colomy (Eds.), Differentiation theory and social change: Comparative and historical perspectives (pp. 409-440). Columbia: Columbia University Press.

Marx, K., y Engels, F. (1972). La ideología alemana. Barcelona: Grijalbo.

Nisbet, R.A. (1973). The Social Bond: An Introduction to the Study of Society. New York: Knopf. 
O'Donnell, G., y Schmitter, P.C. (2013). Transitions from authoritarian rule: Tentative conclusions about uncertain democracies. Baltimore: Johns Hopkins University Press.

O'Donnell, G., Schmitter, P.C., Whitehead, L., y Lowenthal, A.F. (Eds.) (1986). Transitions from Authoritarian Rule (Vols. 1-4). Baltimore: John Hopkins University Press.

Pérez-Agote, A. (2016a). La crise de la représentation démocratique. Le cas espagnol: depuis les indignados jusqu'à Podemos. Socio, 6, 171-200.

Pérez-Agote, A. (2016b). Contemporary Changes in the Processes of Social Differentiation: Towards an Analytical Version of the Theory. En R. Robertson y J. Simpson (Eds.), The Art and Science of Sociology. Essays in Honour of Edward A. Tiryakian (pp.96-117). London-New York-Delhi: Anthem Press.

Ritzer, G. (1992). The McDonaldization of Society. Thousand Oaks: Pine Forge Press.

Ritzer, G. (1996). The McDonaldization thesis: Is expansion inevitable?. International Sociology, $11(3), 291-308$.

Schmitter, P.C. (1986). An Introduction to Southern European Transitions From Authoritarian Rule: Italy, Greece, Portugal, Spain, and Turkey. En G. O'Donnell, P.C. Schmitter, L. Whitehead y A.F. Lowenthal (Eds.), Transitions from Authoritarian Rule (Vols. 1-4) (pp. 3-10). Baltimore: John Hopkins University Press.

Swidler, A. (1986). Culture in action: Symbols and strategies. American Sociological Review, $1986,273-286$.

Thiesse, A.-M. (1999). La création des identités nationales. Europe xvIII ${ }^{e-x x^{e}}$ siècle. París: Seuil.

Tilly, C. (1990). Coercion, Capital, and European States, AD 990-1990. Cambridge, Mass.: Basil Blackwell.

Tiryakian, E.A. (1989). Nacionalismo, modernidad y sociología. En A. Pérez-Agote (Ed.), Sociología del nacionalismo (pp. 143-161). Leioa: Servicio Editorial de la Universidad del País Vasco.

Voegelin, E. (1968/2006). La nueva ciencia de la política: una introducción. Madrid: Katz Editores.

Weber, M. (1969). Economía y sociedad. Ciudad de México: Fondo de Cultura Económica.

Wieviorka, M. (1998). Le multiculturalisme est-il la réponse? Cahiers Internationaux de Sociologie, 105, 233-260. 